intelligence quotient (VIQ) was $87+/-19$ SD) and the mean performance quotient (PIQ) was 76 +/- 17.5. The Purdue Pegboard test results measuring hand skills correlated with IQ scores. A low VIQ was associated with impaired hand skills $(\mathrm{p}<0.0001)$ and preoperative hydrocephalus $(\mathrm{p}=0.02)$, whereas a low PIQ was associated with impaired hand skills $(p<0.0001)$ and incision of the vermis $(p=0.02)$. Impaired hand skills were associated with postoperative cerebellar mutism, oculomotor deficits, cerebellar syndrome, and the need for rehabilitation therapy. (Grill J, Viguier D, Kieffer V et al. Critical risk factors for intellectual impairment in children with posterior fossa tumors: the role of cerebellar damage. $\mathbf{J}$ Neurosurg (Pediatrics 2) Nov 2004;101:152-158). (Reprints: Jacques Grill MD PhD, Department de Cancerologie de l'Enfant et de l'Adolescent, Institut Gustave Roussy, 39-rue Camille Desmoulins, 94805 Villejuif, France).

COMMENT. In this study involving multifaceted therapies for malignant posterior fossa tumors, IQ scores were correlated with preoperative hydrocephalus, incision of the vermis, and degree of postoperative cerebellar damage. A recent study in the Netherlands involving 23 children treated surgically for cerebellar astrocytoma and without additional radio- and chemotherapy, also revealed long-term neurologic, psychological, and behavioral sequelae, a high percentage requiring special education. (Aarsen MA et al. Neurology 2004;62:1311-1316; Ped Neur Briefs May 2004;18:39-40). In children following cerebellar tumor resection, with or without radio- or chemotherapy, a cerebellar cognitive affective syndrome with persistent deficits is a significant complication.

\title{
VENOUS THROMBOSIS IN CHILDREN WITH BRAIN TUMORS
}

The incidence and significance of central venous access device (CVAD) dysfunction and symptomatic thrombosis were determined by retrospective review of 253 consecutive children diagnosed and treated for brain tumors at St Jude Children's Research Hospital, Memphis, TN, and Cleveland Clinic, OH. Symptoms of venous thromboses were pain and swelling of an extremity, chest pain, acute dyspnea, and CVAD dysfunction with need to instill a fibrinolytic agent to restore patency. Chemotherapy was used in $75 \%$ of patients and radiation in $66 \%$. CVAD dysfunction was reported in 54 of $190(28 \%)$ patients with central lines, and thrombotic occlusion was confirmed in 17. Major venous thromboembolic events (VTE) occurred in 6 patients with an episode rate of $2.8 \%$. Major thrombosis was more likely with CVAD dysfunction than without, and CVAD dysfunction was associated with reduced survival rate. (Deitcher SR, Gajjar A, Kun L, Heideman RL. Clinically evident venous thromboembolic events in children with brain tumors. J Pediatr Dec 2004;145:848-850). (Reprints: Steven R Deitcher MD, 675 Almanor Ave, Sunnyvale, CA94085).

COMMENT. CVAD dysfunction and thrombotic occlusion are common complications of brain tumors in children and are risk factors for major VTE involving the upper extremities and superior vena cava. CVAD dysfunction is associated with a poor prognosis, and VTE prevention is recommended in high-risk patients. 